\title{
INTEGRATION OF MANAGEMENT AND SimUlation FOR MANUFACTURING SYSTEMS
}

\author{
TAKAKUWA, S.
}

Abstract: A framework for integrating a management system including enterprise resource planning and manufacturing execution systems (ERP/MES) for a manufacturing system on the shop floor with an associated simulation model is proposed to improve decision making in the IoT/Industry 4.0 environment. Objectoriented simulation modeling tools may be suitable to build simulation models of a smart factory, because they have intelligent objects that stand for the operators, machines, and material-handling components. In addition, it is useful to perform realtime simulation together with real-time scheduling for process control. In doing this the required data for doing simulation must be obtained from big data collected either by sensors or a cyber-physical system.

Key words: Internet of Things, Smart factory, Cyber-physical system, Real-time simulation, ERP/MES
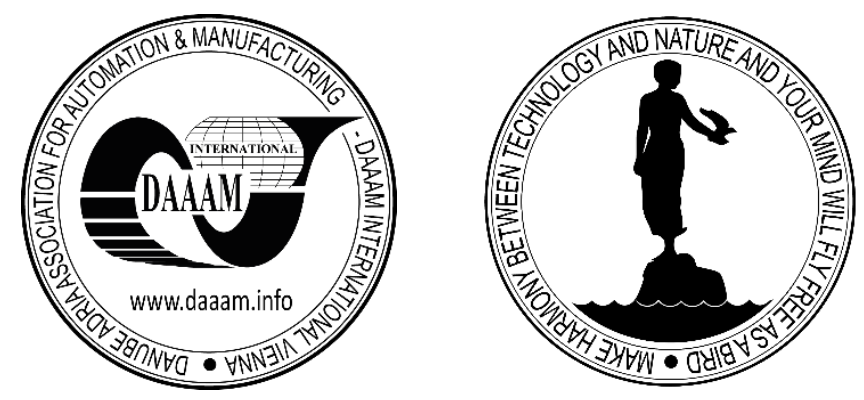

Authors' data: Univ. Prof. Ph.D. Takakuwa, S[oemon]*, *Chuo University, 1-1327, Kasuga, Bunkyo-ku, Tokyo, 112-8551, Japan, takakuwa@indsys.chuo-u.ac.jp

This Publication has to be referred as: Takakuwa, S[oemon] (2018). Integration of Management and Simulation for Manufacturing Systems, Chapter 24 in DAAAM International Scientific Book 2018, pp.273-284, B. Katalinic (Ed.), Published by DAAAM International, ISBN 978-3-902734-19-8, ISSN 1726-9687, Vienna, Austria DOI: $10.2507 /$ daaam.scibook.2018.24 


\section{Introduction}

The fourth industrial revolution or Industry 4.0 is a collective term involving a number of automation, data exchange, and supply chain technologies, including manufacturing systems, via the Internet. In an Industry 4.0 environment, cyberphysical systems communicate with each other and with human participants in real time via the Internet of Things (IoT). Both internal and cross-organizational services are offered and used by members of the supply chain. This allows direct communication between the shop floor and a customer, and mass customization is adopted as a manufacturing system. The concept of mass customization is defined as "producing goods and services to meet individual customer's needs with near mass production efficiency" in both manufacturing and service industries.

In a cyber-physical system, the cyber system can be regarded as a digital twin, because it behaves exactly as the corresponding physical system. A digital twin is used to monitor the current status of the actual manufacturing system and to predict production performance, which explains why simulation plays an important role not only to reflect exactly the current status of manufacturing activity but also to properly predict the expected status of the manufacturing activities.

The IoT/Industry 4.0 environment requires quick decision making, not only by monitoring the status of manufacturing activities but also by administering achievement control. This paper proposes a framework to integrate a management system including enterprise resource planning and manufacturing execution systems (ERP/MES) for a manufacturing system on the shop floor with the associated simulation model to improve decision making in the IoT/Industry 4.0 environment. Next, we stress that simulation and scheduling techniques are needed to make proper real-time decisions, especially from the standpoint of process control in production management. In particular, real-time simulation and risk-based planning and scheduling (RPS) have been described to more effectively control production, especially in the IoT/Industry 4.0 environment.

\section{Shop-Floor Management in Smart Factory}

\subsection{Smart Factory}

A smart factory in the Industry 4.0 environment comprises construction components that identify themselves and recognize their roles in the system, and constitutes an autonomous network. The smart factory is driven by four fundamental design principles (Pegden, 2017):

1) Interoperability of components and data. Machines, devices, sensors, and people connect and communicate with each other via the IoT.

2) Information transparency. Real-time sensor data is connected to the virtual factory model.

3) Technical assistance. Decision-support systems solve urgent problems and the aggregations and visualization of data supports continuous improvement in the manufacturing process. 
4) Decentralized decisions. System components can perform as many autonomous tasks as possible.

\subsection{Digital Twin}

In the 1990s, the concept of the virtual factory had attracted attention, and operators were able to remotely operate real automated factories. Today, "digital twin" is referred to as the consecutive procedure that includes modeling real factories or products as models and simulating these models of the real systems like a twin. In this sense, the way of thinking of the digital twin is very important for manufacturing activities in Industry 4.0. The following sections examine real-time simulation and real-time scheduling, taking the digital twin of real manufacturing systems into consideration.

\subsection{Control of Shop Floor in Terms of ERP/MES}

Recently, the environment of corporate activities changed drastically, partially because of globalization of business, the extreme variability of customer satisfaction, and rapid innovation. Mass customization aims to adopt mass-production processes and satisfy individual customization. Enterprise resource planning (ERP) was proposed to optimize all activities of manufacturers, including logistics management of procurement, manufacturing, inventory, and sales, in addition to financial accounting, management accounting, and personnel management in the 1990s.

ERP consists of two integrations: vertical and horizontal. Horizontal integration is referred to as a supply chain with part suppliers and customers. Conversely, vertical integration comprises management of the ERP system, factory layer, and shop-floor layer inside the corporation. In addition, manufacturing execution systems (MESs) connected to ERP is closely related to shop-floor control.

Fig. 1 shows a framework for integrating the management system including ERP/MES, a manufacturing system on the shop floor, and an associated simulation model. Integration of these three parts should work properly to improve decision making in the IoT/Industry 4.0 environment. In this framework, a real manufacturing system or plant may be replaced by a physical model provided by, say, Fischertechnik or FESTO in a university laboratory.

The planning function of ERP places orders of both parts and materials for suppliers and production instruction, following demand planning or forecasting, master production planning, materials requirement planning. Conversely, production instruction and process progress management for a shop floor are done via MES. In other words, the purpose of MES is to properly use operators, manufacturing equipment, and items. Production instruction is done from ERP to MES, and then MES receives the resulting information on operations from the shop floor. Finally operations achievement is reported to ERP by MES. Production planning, execution, and the associated utilization forms of ERP and MES are summarized in Fig. 2 (SAP, 2014). 
Takakuwa, S.: Integration of Management and Simulation for Manufacturing Syste...

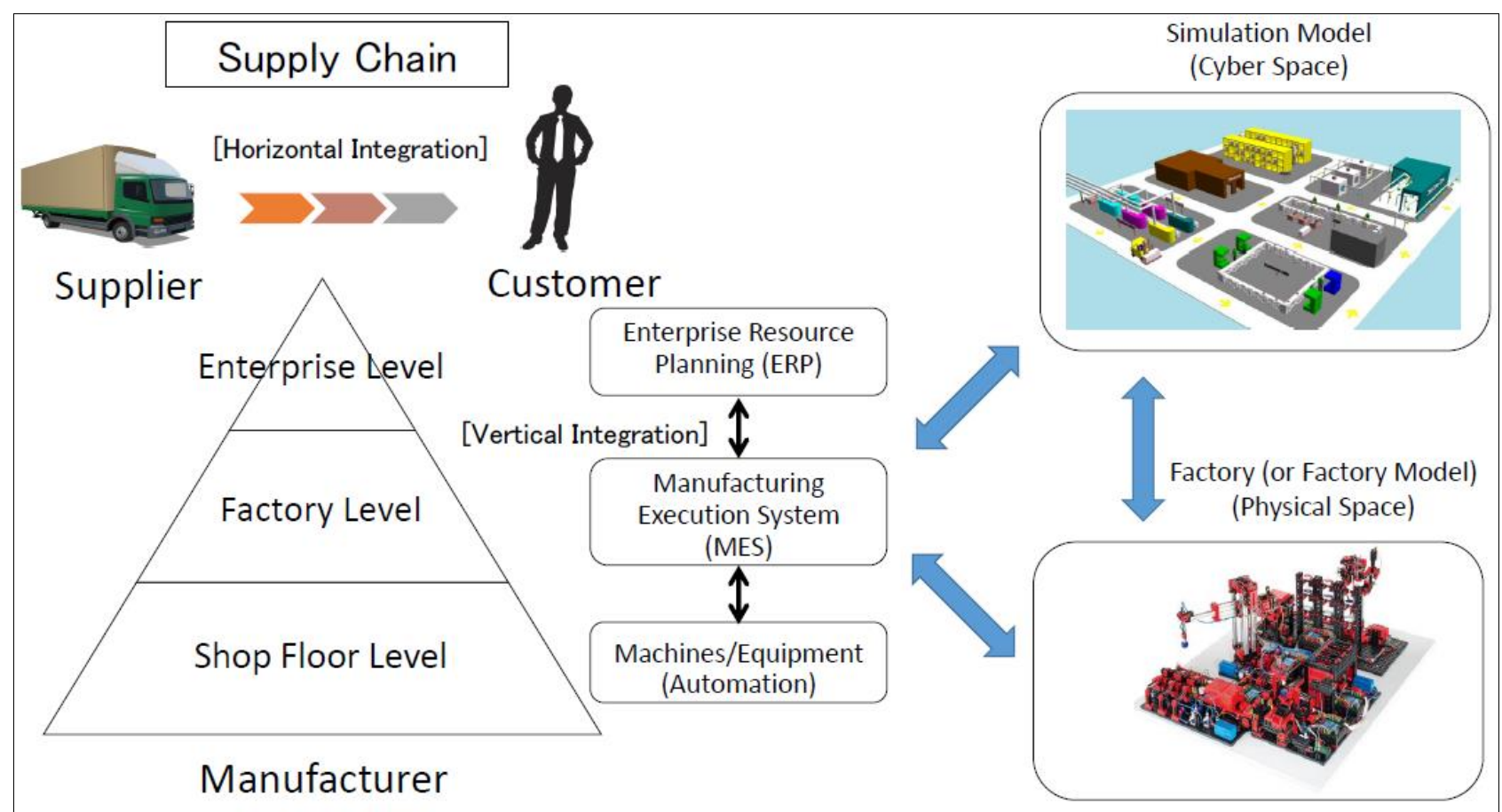

Fig. 1. Framework of management and simulation of manufacturing system.

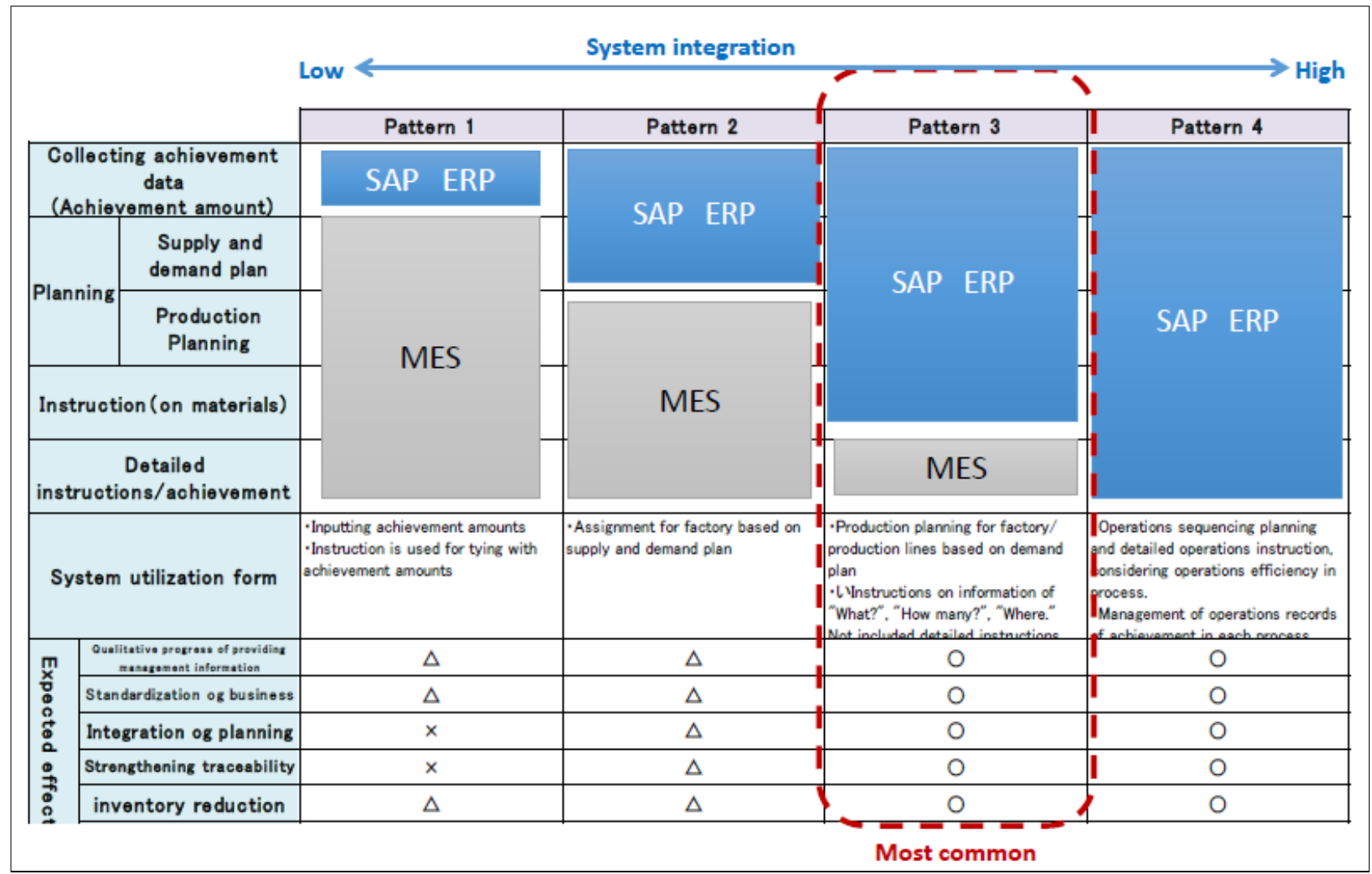

Fig. 2. Division of roles of between ERP and MES (@ SAP Japan Co., Ltd.).

\section{Simulation of IoT/Industry 4.0 environment}

\subsection{Object-oriented simulation}

The U.S. government once announced that simulation technology has become a critical technology for the 21 st century. 
Simulation is regarded as one of the most used management science tools, especially for designing and modifying large-scale and complicated systems, such as manufacturing systems.

Since the late 1950s, simulation models have been built in available languages such as FORTRAN. After the late 1970s, personal computers were becoming faster and cheaper, and special-purpose simulation languages, such as GPSS, SLAM, and SIMAN, were released for modeling logistics, health care, and services and manufacturing systems. At this stage, in process-oriented simulation modeling, in which a sequence of actions occur over time, an entity of a part in a manufacturing system seizes a worker, delays for service, and then releases the worker. A processoriented simulator is very effective and is widely used in practice.

After the 2000s, a new paradigm, object-oriented simulation modeling, was released. For example, Simio, has intelligent objects that stand for the operators, machines, and the material-handling components, allowing us to note the system behavior based on their interaction.

In the IoT/Industry 4.0 environment, operators, machines and equipment, robots, and other objects behave autonomously and interact with each other in a manufacturing system. This means that object-oriented simulation modeling tools are more suitable for building the associated simulation models in smart factory, describing the model from the point of view of the facility.

\subsection{Input Data for Real-Time Simulation}

Traditionally, randomness is adopted to do simulation experiments. Distributions such as the triangular distribution and the normal distribution together with the associated parameters are specified as the input of the simulation model, especially for processing time and interarrival time, and so on. Conversely, statistical analysis of the resultant output is done on the designated performance measure (i.e., 95\% confidence interval on) for the expected value of the designated measure.

Since the 1990s, data files (e.g., an Excel file) containing values were used outside simulation models. This means that specified values were used instead of random values sampled from the specified distribution. This method has enabled us to build simulation models of large-scale and complex systems, by using electronic patient records of a general hospital (Takakuwa \& Katagiri, 2007), point-of-sale data of a retail store (Miwa \& Takakuwa, 2008), and so on.

A simulation is a broad collection of methods and applications that mimics the behaviour of a real or physical system. In the context of the cyber-physical system, a simulation is one of the tools to be applied in the cyber system of a digital twin. The corresponding simulation model runs at the same rate as the actual manufacturing system. This simulation is called a real-time simulation, and the simulation model runs at the same pace as the physical system.

After the 1990s, the concept of real-time simulation was introduced. For example, there are two machine tools with WIP storage space that are connected to a PC, and the actual data on subjects such as machining and setup operations are sent from the corresponding machine tools to the simulation model of the PC. Fig. 3 shows an example of a real-time simulation. In this figure, the real-time console appears on the 
left side of the screen (Drake \& Smith, 1996). Decision are made when processing machining orders, considering evaluation criteria. The status on the shop floor is transmitted to the PC, and the corresponding simulation model in the PC reflects and represents the exact situation of the shop floor. After that, consequences can be foreseen by performing real-time simulations. The simulation model was written in Arena (Kelton, et al., 2015).

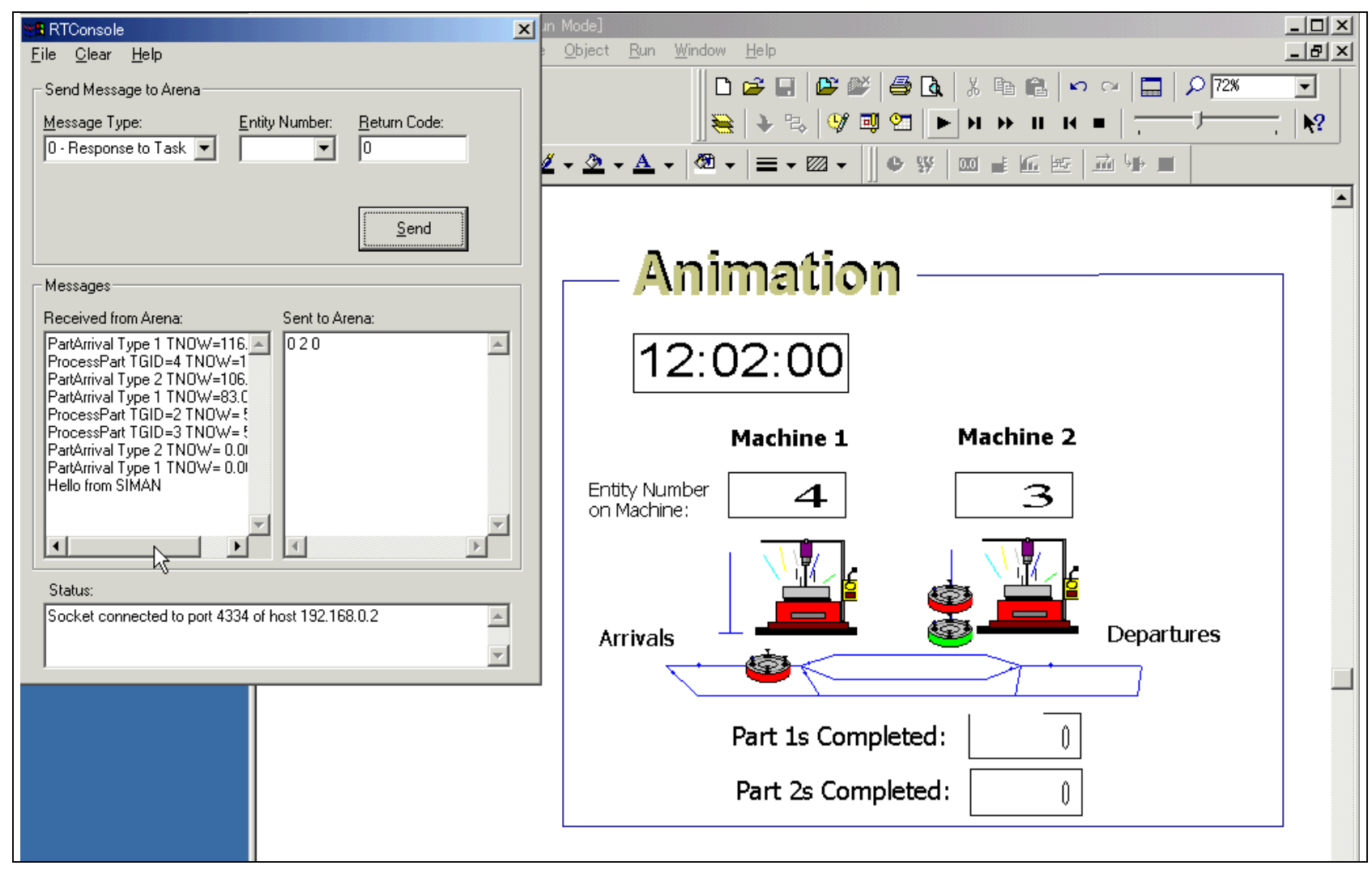

Fig. 3. Real-time simulation.

A simulation model was constructed to reflect the physical system, and simulation experiments are carried out, by using a distributed model equipped with a sensor as the physical system, (Yang, et al., 2017). By attaching a series of sensors to the designated locations of the manufacturing system, the data thus collected are sent to a PC to execute an associated simulation model (Yoshida, 2018). In this case, a factory model comprises three subsystems, i.e., AS/RS, sorting line, and multi-processing station. A work piece is first routed to the multi-processing station.

After processing, the work piece is transferred on the conveyor to the sorting line. Nine work pieces are processed as one cycle of operations. Optical sensor and ultrasonic distance sensors are facilitated in the factory model. All sensors are connected to a microcomputer. Each sensor is assigned an individual number for identification. Associated data is stored in an Excel file. Arena simulation software is used in conjunction with Excel files. Fig.4 shows an overall picture of a physical manufacturing system or a factory model and simulation and animation model together with an Excel file. 


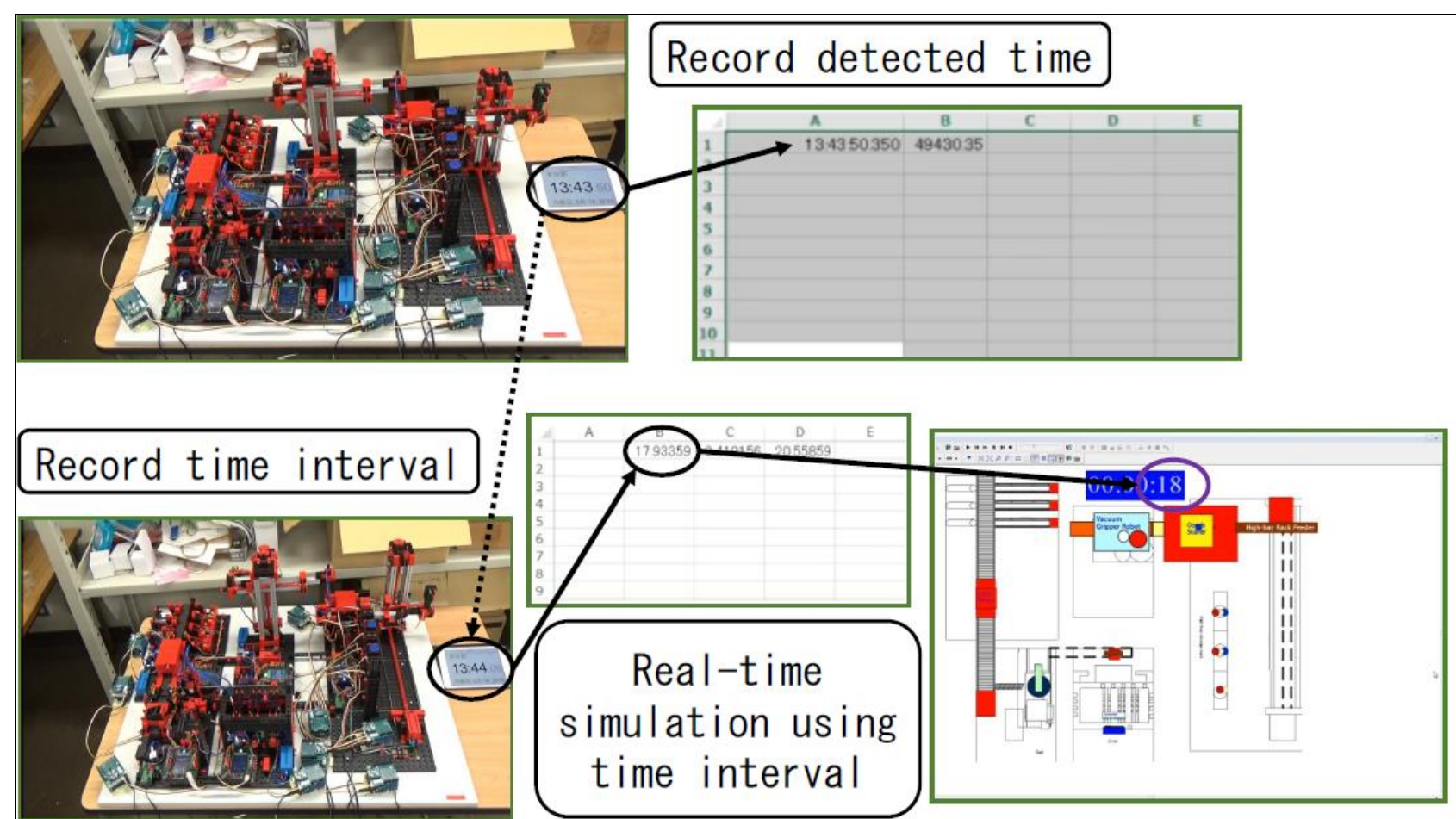

Fig. 4. Real-time simulation using IoT (Yoshida, 2018).

When the sensor detects an object, the associated signal is sent to a microcomputer, which checks the designated action from the signal. If appropriate, it sends the sensor number to a PC. Receiving time is recorded in the Excel file, and time interval is recorded when the sensor detects the following object.

The Arena system refers to the recorded time in the Excel file, and the simulation is executed. Fig. 5 shows the relation between a sensor, a microcomputer, and a PC. When a sensor in AS/RS detects an object, detection time is recorded in the Excel file. Next, the second sensor detects an object, and the time interval is recorded in another worksheet. Simulation execution is suspended while no data appear in the Excel file; simulation execution resumes when at least one datum appears. In addition, simulation time in animation reflects the data of the Excel file.

Simulation models of an automotive flow shop production were automatically created from the company's SAP ERP and MES system to support operational planning and to reduce operational logistical risks (Kirchhof, 2016). In addition, an approach on how to connect between engineering of machine tools and an engineering using cyberphysical systems was proposed (Scheifele, et al., 2017).

A real-time simulation as a digital twin will be increasingly used in the future in the IoT/Industry 4.0 environment. Data of the real (physical) manufacturing systems are extracted either via sensors or directly from the manufacturing system itself. Therefore, it is important to pick up the required data for executing simulations based on big data that are collected either via sensors or via a cyber-physical system. 
Takakuwa, S.: Integration of Management and Simulation for Manufacturing Syste...

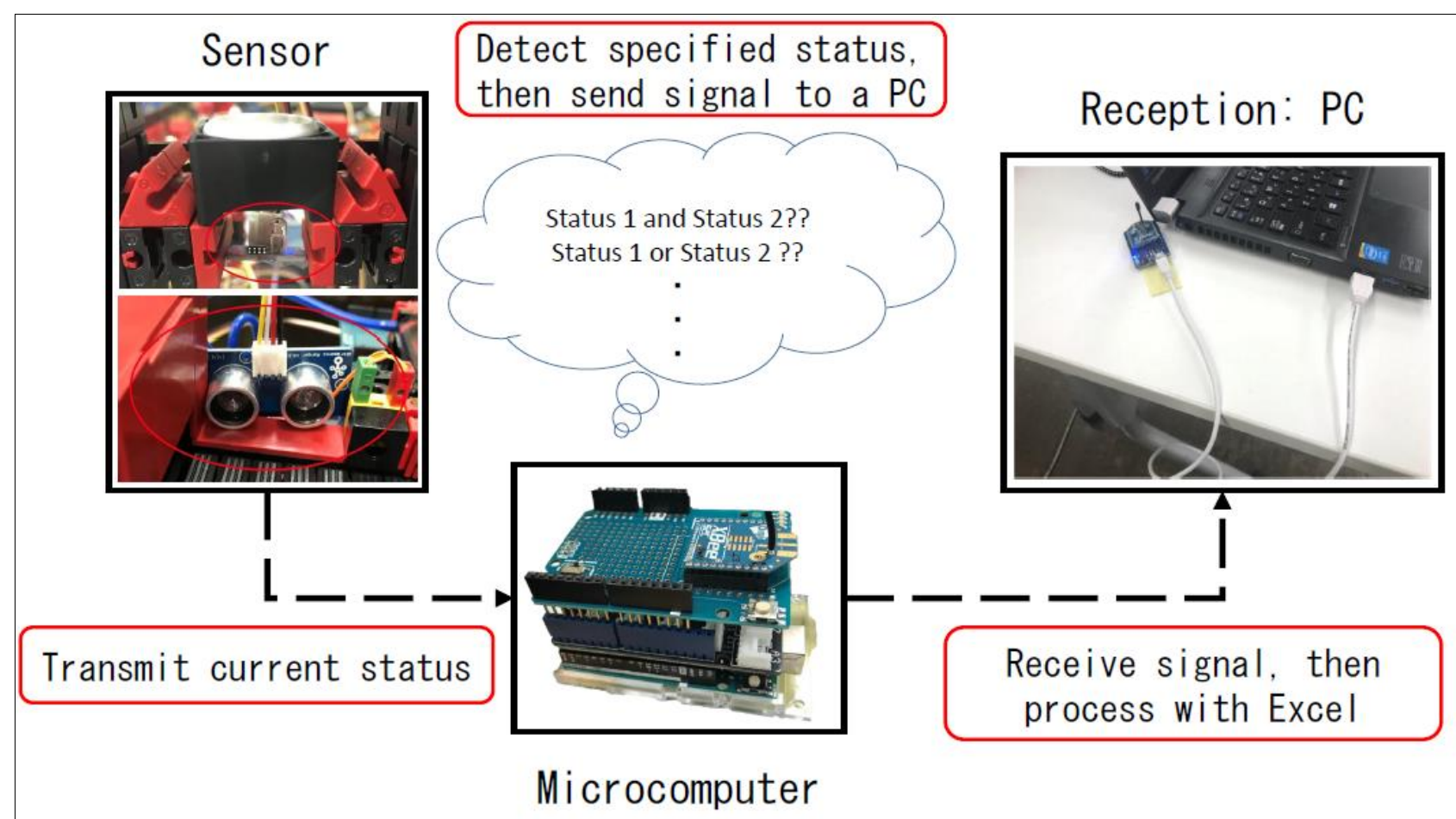

Fig. 5. Data gathering via sensors (Yoshida, 2018).

\section{Real-time Scheduling}

\subsection{Scheduling as a Process-Management Tool}

The operation or production means to create utility or increase the value of products or services are outputs generated through activities of conversion from inputs called factors of production which consist of materials, production labor, machines and/or equipment, and production information (Hitomi, 1979). The value of a product or service can be determined from aspects of the value, including function and quality, production cost and price, production quantity and time or due date, and environment.

Process control comprises two functions of planning and control in a physical process (Takakuwa, 2015). One of the major objectives of process control is to deliver the items by the agreed date, namely; the due date. However, expected but unplanned interruptions cause delays and inefficiencies for manufacturing activities; for example, machines might break down, workers might be absent, or materials might arrive late.

By installing the IoT, the plant monitoring system would improve the responsiveness of a factory to unplanned interruptions. It reduces delays and inefficiency due to minute-to-minute plant floor disturbances. The key to improved factory responsiveness is faster and more effective shop-floor communication. Although monitoring is essential to shop floor control, it cannot cope with achievement control. Monitoring itself cannot provide the exact solutions for these what-if type of questions. In the following section, the current status of the development on simulation and scheduling techniques are briefly introduced to control achievement in the IoT and Industry 4.0 environments. 


\subsection{Simulation and Scheduling}

Discrete event simulation (DES) can be used for finite-capacity scheduling. The quality of the simulation based scheduling is determined based on the decision logic. A typical architecture for using DES is illustrated in Fig. 6 in conjunction with the ERP system and MES.

However, most DES is difficult to initialize the model of the current state of the physical system because the initial status of the scheduling model is "empty and idle." Therefore, RPS is proposed as one of approaches to resolve this shortcoming, combining deterministic and stochastic simulation (Kelton, et al., 2017).

Thus, a management system including ERP/MES, a manufacturing system at a shop floor, and simulation should be integrated to achieve effectively process management, especially in the IoT/Industry 4.0 environment.

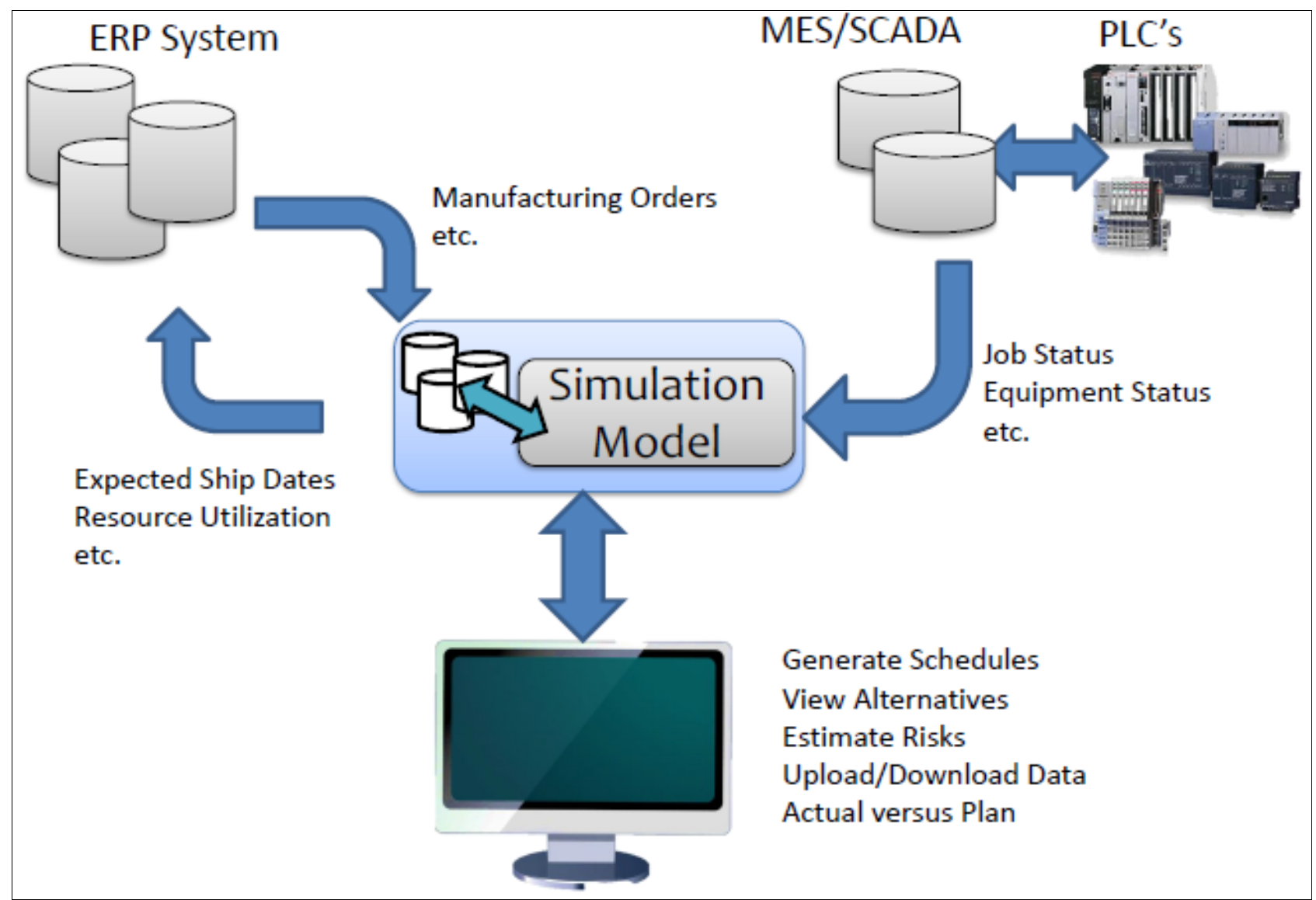

Fig. 6. Architecture of a typical simulation-based scheduling system (Kelton, et al., 2017)

\subsection{Risk-Based Scheduling}

In this section, the scheduling approach of RPS is described to consider the risk of tardiness of job completion time. In a smart factory, the following insights are obtained, by constructing the virtual factory model with the ERP and MES in conjunction with IoT (Pegden, 2017):

- Visualize the system to gain insights.

- Optimize future use of assets to meet key performance indicators.

- Support "what if" analysis to test ideas. 
- Assess and mitigate schedule risk.

- Set future data patterns and trends.

- See and address future bottlenecks.

Such a simulation and scheduling model represents a potential tool to help the manufacturer decide in the real time by further connecting the model to the MES and ERP system.

As an example, a manufacturing system which comprises cut, weld, shape, and finish processes is considered to illustrate an application of RPS. One or two machines are installed at each process. If multiple machines are available at a process, selection is made based on a specified scheduling rule. In addition, two types of parts are processed according to operation sequence, setup time, and processing time. Fig. 7 shows a sample of the work flow of orders, including the high risk of being late. Degree of satisfaction and violation to meet the associated due date are represented in percentage for each order; thus, risk analysis is very effective, especially after receiving an unexpected or sudden order while operating at the shop floor. For process control, it is useful to perform simulation together with real-time scheduling in the IoT/Industry 4.0 environment (Yang \& Takakuwa, 2017).

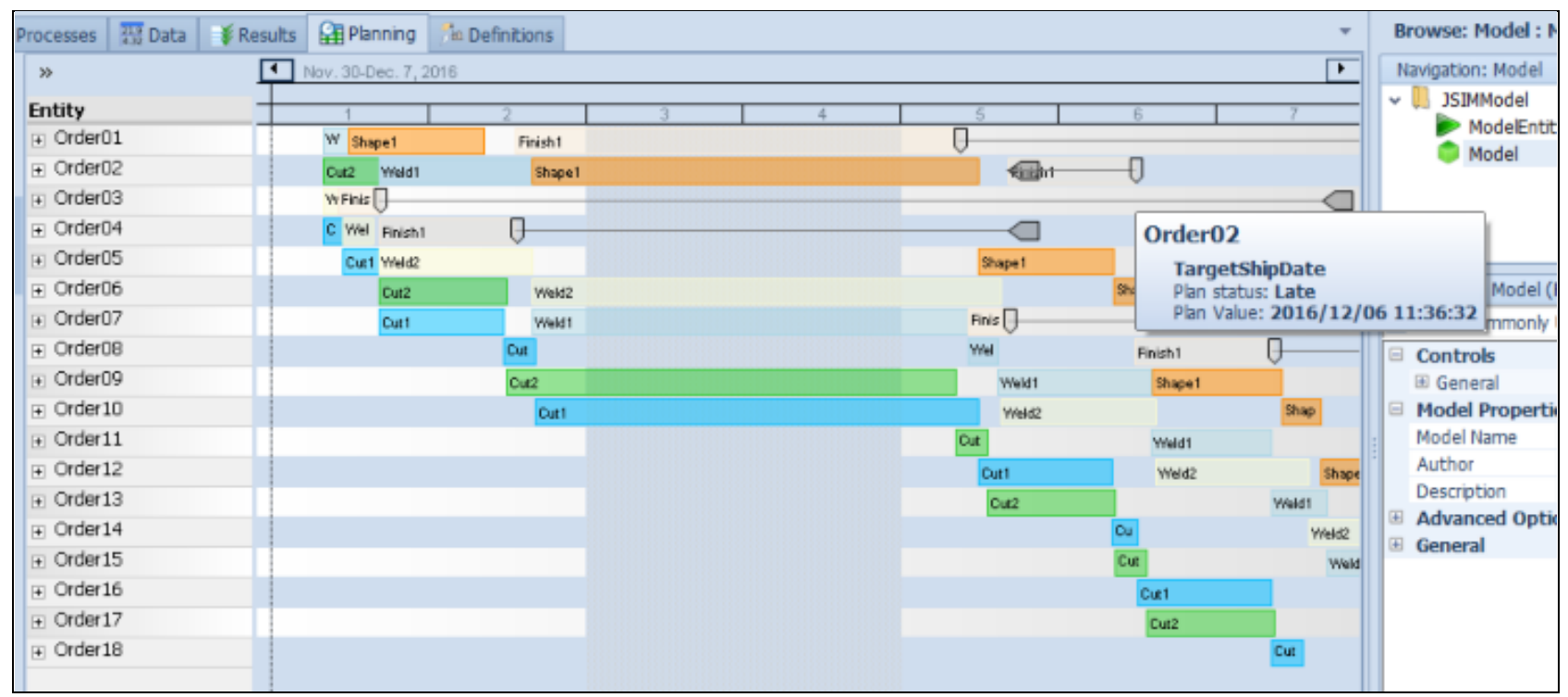

Fig. 7. Sample of work flow of orders in Simio real-time scheduling.

\section{Conclusions}

The following conclusions may be extracted from this work:

1) In the IoT/Industry 4.0 environment, integration of a management system of ERP/MES considering a supply chain, a manufacturing system in the shop floor, and the associated simulation model is essential to improve decision making.

2) A smart factory comprises construction components, such as machines and materials-handling equipment that identify themselves and recognize their roles in the system, and constitute an autonomous network. Object-oriented simulation modeling tools may be suitable to build simulation models of a smart factory, because it contains 
intelligent objects that represent operators, machines, and the material-handling components.

3) Traditionally, simulations were used mainly for design or improvement of manufacturing systems. It is useful for process control to perform simulations together with real-time scheduling in IoT/Industry 4.0 environment. From the standpoint of operations management, achievement control in process control is treated to implement shop floor control, and to perform expeditious follow-up and control of available capacity to adjust for the difference between the planned amount and the actual amount. Simulation and scheduling techniques will play an important role in meeting deadlines in the process control and the accounting of ERP/MES and shop data. In this context, real-time simulation and risk-based planning and scheduling were briefly presented.

4) Traditionally, randomness is adopted to execute simulation experiment. In the context of the cyber-physical system, a simulation can be considered as one of the tools to apply in the cyber system of a digital twin. In a real-time simulation, it is important to pick up the required data for executing simulations based on big data that are collected either via sensors or via a cyber-physical system.

5) As shown in Fig. 1, a framework for integrating the management system including ERP/MES, a manufacturing system on the shop floor, and an associated simulation model. Integration of these three parts is proposed to improve decision making in the IoT/Industry 4.0 environment. Three links between ERP/MES and a manufacturing system, between a manufacturing system and an associated simulation model, and between ERP/MES and an associated simulation model should be constructed to fully realize the proposed framework. Some of them are still future tasks.

\section{Acknowledgments}

This research work was supported by Chuo University Joint Research Grant, "Paradigm shift of the Manufacturing Business in IoT/Industry 4.0 Environment in East Asia."

\section{References}

Drake, G.R. \& Smith, J. S. (1996). Simulation system for real-time planning, scheduling, and control, Proceedings of the 1996 Winter Simulation Conference, Charnes, J. M.; Morrice, D. J., Brunner, D. T., \& Swain, J. J. (Ed.), IEEE, 1083-1090. https://www.informs-sim.org/wsc96papers/155.pdf. Accessed: 2018-9-20.

Hitomi, K. (1979). Manufacturing Systems Engineering, Taylor \& Francis, London. ISBN 0-85066-177-3.

Kelton, W. D.; Sadowski, R. P. \& Zupick, N. B. (2015). Simulation with Arena, Sixth Edition, McGraw-Hill. New York, NY. ISBN: 0073401315.

Kelton, W. D.; Smith, J. S. \& Sturrock, D. T. (2017). Simio and Simulation: Modeling, Analysis, Applications. Fourth Edition, Simio LLC. Sewickley, PA. ISBN: 9781542933117.

Kirchhof, P. (2016). Automatically generating flow shop simulation models from SAP data, Proceedings of the 2016 Winter Simulation Conference, Roeder, T. M. K., 
Frazier, P. I., Szechtman, R., Zhou, E., Huschka, T., and Chick, S. E. (Ed.), IEEE, 3588-3589. https://www.informs-sim.org/wsc16papers/335.pdf. Accessed: 2018-9-20. Miwa, K, \& Takakuwa, S. (2008). Simulation modeling and analysis for in-store merchandizing of retail store with enhanced information technology, Proceedings of the 2008 Winter Simulation Conference, Mason, S. J., Hill, R. R., Monch, L., Rose, O., Jefferson, T., and Fowler, J. W. (Ed.), IEEE, 1702-1710.

http://www.informs-sim.org/wsc08papers/209.pdf. Accessed: 2018-9-20.

Pegden, C.D. (2017). Deliver on Your Promise: How Simulation-Based Scheduling Will Change Your Business, Simio LLC, Sewickley, PA.

SAP. (2014). SAP Blog: Difference of rols between ERP and MES. https://www.sapjp.com/blog/archives/6480. Accessed: 2017-12-27. (in Japanese)

Scheifele, S.; Riedel, O. \& Pritschow, G. (2017). Engineering of machine tools and manufacturing systems using cyber-physical systems, Proceedings of the 2017 Winter Simulation Conference, Chan, W. K. V., D’Ambrogio, A., Zacharewicz, G., Mustafee, N., Wainer, G., and Page, E. (Ed.), IEEE, 1503-1514.

https://www.informs-sim.org/wsc17papers/includes/files/118.pdf. Accessed: 2018-920.

Takakuwa S. (2015). Operations management, Chuo-Keizaisha, Tokyo, ISBN 978-4502-14311-3. (in Japanese)

Takakuwa, S. \& Katagiri, D. (2007). Modeling of patient flows in a large-scale outpatient hospital ward by making use of electronic medical records, Proceedings of 2007 Winter Simulation Conference, Henderson, S.G., Biller, B., Hsieh, M.-H., Shortle, J., Tew, J. D., and Barton, R. R. (Ed.), IEEE, 1523-1531.

http://www.informs-sim.org/wsc07papers/185.pdf. Accessed: 2018-9-20.

Yang, W. \& Takakuwa, S. (2017). Simulation-based dynamic shop floor scheduling for a flexible manufacturing system in the Industry 4.0 environment, Proceedings of the 2017 Winter Simulation Conference, Chan, W. K. V., D'Ambrogio, A., Zacharewicz, G., Mustafee, N., Wainer, G., and Page, E. (Ed.), IEEE, 3908-3916. https://www.informs-sim.org/wsc17papers/includes/files/330.pdf. Accessed: 2018-920.

Yang, W.; Tan, Y., Yoshida, K., \& Takakuwa, S. (2017). Digital Twin-driven Simulation for a Cyber-physical System in Industry 4.0 Era, DAAAM Scientific Book 2017, Katalinic, B. (Ed.), DAAAM International, Vienna, Austria, 227-234. ISBN 978-3-902734-12-9, ISSN 1726-9687. DOI: 10.2507/daaam.scibook.2017.18.

Yoshida, K. (2018). Designing and constructing IoT recording system for real-time simulation, Proceedings of 76th JSIM Conference, Japan Society for Information and Management, Tokyo, 221-224. (in Japanese) 